\title{
Competencias investigativas en docentes de la asignatura de investigación de educación básica y media
}

\author{
Research skill in teachers of the research subject of basic and high school education
}

\author{
a- Lyda Lorena Barón Pinto \\ lorena.baronp@gmail.com \\ Código ORCID: 0000-0002-5010-6706 \\ Universidad Metropolitana de Educación, Ciencia, Investigación y Tecnología, Panamá
}

Artículo recibido en octubre 2019 | Arbitrado en noviembre 2019 | Publicado en enero 2020

\begin{abstract}
Resumen
El objetivo de la investigación fue describir las competencias investigativas que poseen los docentes de la asignatura de investigación de educación básica y media, de las instituciones educativas de Sogamoso - Boyacá. La investigación fue descriptiva, con un diseño de campo, transeccional, contemporáneo. La población estuvo conformada por 38 docentes de educación básica y media que orientan la asignatura de investigación en las instituciones educativas de Sogamoso, Boyacá. Para el análisis de datos se utilizaron frecuencias, porcentajes, y medianas. Los hallazgos evidencian que las competencias investigativas de los docentes y la formación metodológica recibida no son suficientes para desarrollar investigaciones de calidad. La sinergia competencias actitudinales fue la que mejor puntaje obtuvo, la sinergia competencias cognitivas fue la más baja y la sinergia competencias procedimentales fue regular, lo que quiere decir que los docentes, manifiestan una actitud positiva frente a la investigación.
\end{abstract}

\footnotetext{
Abstract

The objective of the research was to describe the investigative competences possessed by the teachers of the basic and secondary education research subject of the educational institutions of Sogamoso - Boyacá. The research was descriptive, with a field, transectional, contemporary design. The population was made up of 38 basic and high school teachers who guide the research subject in the educational institutions of Sogamoso, Boyacá. For data analysis, frequencies, percentages, and medians were used. The findings show that the investigative competences of teachers and the methodological training received are not sufficient to develop quality research. The synergy of attitudinal competencies was the one with the best score, the synergy of cognitive competences was the lowest and the synergy of procedural competences was regular, which means that the teachers show a positive attitude towards the research.
}

Palabras clave:

Investigación; competencias investigativas; docentes investigadores
Keywords:

Research; investigative/research skills; teacherresearcher 
INTRODUCCIÓN

La investigación es un proceso fundamental para el desarrollo de la sociedad. Es tal su impacto que formar a niños y jóvenes en investigación va de la mano con grandes avances sociales. Se ha hecho énfasis en la necesidad de comenzar a desarrollar competencias investigativas desde las primeras etapas de la educación, sin embargo, el tema de la formación de niños, niñas y jóvenes en ciencia, tecnología e investigación científica es relativamente reciente en Colombia. La problemática se caracteriza por el uso de estrategias pedagógicas de muy bajo interés educativo, y los docentes no están adquiriendo las competencias investigativas para orientar a los niños en la cátedra de investigación.

Aun cuando los niños poseen un alto potencial cognitivo para investigar, debido a que es innata su curiosidad y su deseo de conocer el mundo a partir de preguntas, es frecuente que esas habilidades no se desarrollen para convertirse en competencias de investigación, pues los docentes encargados de potenciar estas habilidades de los niños, tampoco han logrado, a lo largo de su formación, desarrollar competencias metodológicas y científicas.

El desarrollo de las competencias de investigación es hoy en día una tarea prioritaria en la formación de los nuevos profesionales, sin embargo, estas competencias son variadas y complejas, por lo que no se logran a corto plazo, sino que requieren de un proceso progresivo que debe iniciar en las primeras etapas de la educación. Por ello es urgente promover la formación metodológica de los docentes, a partir de una didáctica apropiada, que los ayude a incorporar, en su labor cotidiana dentro del aula, las actividades que los niños requieren para desarrollar, en concordancia con su proceso evolutivo, las competencias que se requieren para que puedan convertirse, a futuro, en hábiles investigadores.

Esta investigación abordó precisamente la problemática de las competencias investigativas de los docentes, con el fin de dar aportes para comprender mejor la situación y aportar algunas soluciones.

En el marco de la problemática estudiada se destacó lo que Hurtado de Barrera (1999) explica acerca de la investigación, pues esta constituye una actividad permanente dentro del proceso evolutivo de la humanidad, que procede de la inquietud propia del ser humano por conocer su entorno y conocerse a sí mismo. Como parte de este proceso, la investigación es indispensable en el avance de los pueblos, pues de ella deriva el conocimiento y la tecnología que le permiten al ser humano mejorar cada vez más sus condiciones de vida. Sin embargo, CEPAL (2004) considera que América Latina y el Caribe aún se encuentran muy rezagadas en comparación con otras regiones del mundo en términos de investigación y desarrollo. Mientras Estados Unidos, Japón y la República de Corea invierten en este rubro entre 2,5 y 3 puntos de su producto interno bruto (PIB) y la Unión Europea cerca de 2 puntos, nuestro continente en su conjunto destina apenas 0,5 puntos del PIB.

Según la Red de indicadores de ciencia y tecnología RICYT (2018), los países latinoamericanos representan aproximadamente el $2 \%$ de la inversión mundial en investigación y desarrollo (I+D), muy lejos de Norteamérica (39\%), Europa (31\%) y Asia (26\%). Brasil es el país que más invierte en I+D $(54 \%$ de la inversión latinoamericana), seguido de México con 
$26 \%$. Se ha señalado que las capacidades para llevar a cabo actividades de investigación y desarrollo de un país están representadas fundamentalmente en la cantidad y calidad de investigadores que posee, en los grupos de investigación, y en los productos generados por esos grupos de investigación Colciencias (2017). Para Castillo (1999) una de las principales causas del escaso desarrollo científico-tecnológico de Colombia es la reducida comunidad científica, este país sólo aporta el 1\% de los investigadores de Latinoamérica y el $0.01 \%$ del total mundial.

En el caso de Colombia, de acuerdo a Colciencias (2017), la política nacional en CTeI (Ciencia, tecnología e innovación) reconoce la importancia de la investigación para expandir la frontera del conocimiento y como fuente de trasformaciones sociales, económicas y ambientales. Para Restrepo (2004), la mejor forma de construir la cultura investigativa es a través de la promoción de investigadores prominentes que cultivan sus líneas de investigación y reúnen en torno a su trabajo a estudiantes aventajados.

La educación tiene, entonces, una importante misión en lo que respecta a la investigación. La finalidad de la educación es preparar al ser humano dentro de un contexto de creatividad, sentido crítico y conciencia social, para la percepción amplia del mundo y para el dominio de los medios necesarios en su transformación, tal como los cambios actuales lo exigen. En este sentido, se ha hecho hincapié en la necesidad de integración de la ciencia a todos los niveles de la vida social, como un medio para sensibilizar a las nuevas generaciones en torno a una apreciación científica de la realidad (UNESCO, 1990 citado por Hurtado de Barrera, 1999).
Sin embargo, esta formación temprana relacionada con las competencias investigativas no se está logrando. Zamora (2014) señala que los estudiantes presentan dificultades para apropiarse del conocimiento y sobre todo para desarrollar las habilidades investigativas; es así que en la mayoría de los casos las actividades que realizan no logran ser aplicadas en la práctica y peor aún, lo aprehendido no puede ser transferido a nuevas situaciones del entorno social. Este autor considera que las tareas ayudan a los estudiantes a utilizar el conocimiento de manera significativa e incluyen tres características: tareas a largo plazo, multidimensionales, y dirigidas por el alumno. Para lograr el propósito antes señalado, los docentes tienen que comenzar por saber investigar, pues no se puede enseñar lo que no se sabe. Sin embargo, son pocos los profesionales, incluso los docentes, que han logrado formarse como investigadores.

La investigación pedagógica se logra cuando el profesor indaga, entiende los problemas de su práctica cotidiana, genera hipótesis de trabajo y desarrolla sus temas con base en preguntas. La innovación pedagógica responde a la manera como los maestros inciden en la transformación de la cultura escolar desde la formación temprana, introducen cambios en las prácticas tradicionales, y mejoran la calidad y las formas de aprender en las escuelas (Gutiérrez, 2014). La mayoría de los docentes no consideran que a través de las diversas asignaturas se puede iniciar un proceso de aprendizaje investigativo. Para Zamora (2014) existe la necesidad de que el docente enseñe al estudiante cómo investigar por medio de técnicas y herramientas pertinentes en cada una de sus actividades académicas. Esto implica el 
desarrollo de competencias, tanto generales como específicas, de investigación por parte del docente.

De acuerdo con la problemática expuesta, se planteó la siguiente pregunta de investigación: ¿Cuáles son las competencias investigativas que poseen los docentes de la asignatura de investigación de educación básica y media, de las instituciones educativas de Sogamoso - Boyacá?

\section{Competencias: Nociones y características}

Para formar personas competentes es preciso que las instituciones educativas implementen procesos pedagógicos y didácticos fundamentados en competencias, los principales responsables son los docentes y las autoridades educativas, aunque la formación de competencias no es responsabilidad exclusiva de las instituciones educativas (Contreras, 2014).

Tobón (2007) considera que las competencias son pensadas como procesos que construyen, reconstruyen y afianzan las personas con el fin de comprender, analizar y resolver diferentes tareas $y$ problemas de los entornos laborales, con conciencia reflexiva, autonomía y creatividad, buscan el crecimiento de la productividad de la organización desde la propia autorrealización personal, emplean de forma racional los recursos ambientales disponibles y tienen en cuenta la complejidad e incertidumbre de la situación.

El MEN de Colombia (2006) define competencia como el "saber hacer en situaciones concretas que requieren la aplicación creativa, flexible y responsable de conocimientos, habilidades y actitudes" (p. 37). También se consideran como el conjunto de habilidades para movilizar conocimientos, reflexionar sobre la acción y estar dispuesto a afrontar un proceso, a partir de una serie de rasgos y atributos personales, como conocimientos, destrezas y actitudes que forman parte del dominio de la persona, y le permiten desempeñarse en un área del saber (MEN, 2010). Ser competente es saber hacer y saber actuar con base en un conocimiento, expresado en valores y principios, con el objetivo de que los estudiantes se apropien de sus talentos, potencialidades y resuelvan problemas en un determinado contexto a partir de un aprendizaje significativo, autónomo y activo. La competencia además de ser un saber hacer, es un saber haciendo, basado en vivencias y conocimientos que las personas adquieren en el transcurso de la vida; es la utilización flexible e inteligente de los conocimientos que se tienen, lo que hace competente al ser humano frente a tareas específicas.

El Proyecto PISA (2006 como se citó en Quiroga et al, 2014) define la competencia como la capacidad de emplear los conocimientos de una persona y el uso de ese conocimiento para identificar problemas, adquirir nuevos conocimientos, explicar fenómenos científicos y extraer conclusiones basadas en pruebas sobre cuestiones relacionadas con la ciencia. Sin embargo, para Pérez (2012), el desarrollo de competencias investigativas implica saber utilizar el conocimiento, afianzar habilidades para observar, preguntar, argumentar, sistematizar, con el objetivo de crear o gestionar el conocimiento, sobre la base del interés, la motivación hacia la investigación, el desarrollo de sus capacidades y la realización personal del estudiante.

Las competencias se observan por medio de los desempeños y actuaciones que ellos realizan en situaciones cotidianas $\mathrm{O}$ estructuradas. Para el MEN (2009) las competencias como 'hacer' y 'saber hacer', que se transforman en 'poder hacer', se 
observan en el uso que los niños hacen de los objetos de acuerdo con las prácticas de su entorno. Así, ellos empiezan a tener comportamientos y a realizar actividades que caracterizan a los integrantes de su comunidad, al cumplir un papel fundamental en su proceso de socialización.

Existen diferentes criterios para clasificar las competencias. Se pueden clasificar en función del tipo de habilidad que se requiere (cognitivas, actitudinales y procedimentales), según el nivel de complejidad (generales y específicas), y según la disciplina o el área de desempeño (competencias en biología, en matemáticas, en lengua...).

Las competencias en investigación se entienden como el conjunto de saberes, habilidades y disposiciones de una persona, que le permiten utilizar sus conocimientos, reflexionar sobre la acción, y estar dispuesta a desarrollar un proceso investigativo, a partir de una serie de cualidades personales, que forman parte del dominio de la persona, y le permiten desarrollar una investigación con eficiencia y calidad. En concordancia con lo anterior, las competencias en investigación pueden ser básicas -o generales-, y específicas. Estas competencias, básicas y específicas, también pueden ser de orden cognitivo, actitudinal o procedimental. A partir de la lectura y el análisis de autores como Tobón (2006), Tacca (2011), Pérez (2012), y de los planteamientos del MEN Colombia (2006), se organizó la clasificación de los diferentes tipos de competencias en investigación.

a. Competencias generales cognitivas en investigación:

- Competencias de razonamiento general: reflexión y abstracción, inferencias, deducciones, establecer relaciones causales y formular hipótesis (MEN, 2009; Pérez, 2012).

- Competencias para la lectura, la documentación y la ubicación de información, como la lectura compresiva y crítica, y la consulta de fuentes (Martínez y Orozco, 2002; Leiva, 2013; Aldana de Becerra, 2012).

- Habilidad para detectar situaciones y problemas, atreverse a preguntar, describir contextos y registrar información acerca de situaciones problemáticas. Además, involucra la capacidad de proponer soluciones a problemas detectados (Federman, Quintero y Munévar, 2001; Pérez, 2012; Mendivelso, 2006; Leiva, 2013).

- Competencias de anticipación y planeación: se refieren a la posibilidad de distinguir que los actos tienen consecuencias en los planes; implican la habilidad de realizar inferencias elaborar planes, establecer relaciones causales y formular hipótesis y teorías. La competencia de planificación implica saber organizar el tiempo y los recursos.

- Competencias tecnológicas (Martínez y Orozco, 2002): En general todo profesional y todo investigador requiere de competencias tecnológicas que le permitan manejar diferentes aplicaciones como procesadores de texto, programas de presentación, ilustración y de búsqueda y documentación, así como manejar buscadores de internet, tener conocimiento sobre computadoras y sobre tecnología, representación de procedimientos, uso de procesadores de texto, bases de datos, gráficos, utilización de las telecomunicaciones, 
para colaborar, publicar e interactuar con otros.

- Competencias de cálculo y manejo numérico (Martínez y Orozco, 2002). Esto involucra dominar operaciones matemáticas básicas, saber usar fórmulas y conocer el lenguaje matemático, lo cual es la base para poder utilizar diferentes técnicas de análisis.

b. Competencias generales procedimentales en investigación:

- Competencias comunicativas: Según Uribe (2013 como se citó en Leiva, 2013) las competencias comunicativas son un eje transversal en el proceso de investigación, desde la comunicación verbal asertiva, para que el estudiante se relacione con los otros en la solución de problemas, hasta la producción escrita, donde es necesario repensar ideas y expresarlas para tener espacio de transcribirlas. Mendivelso (2006), considera que dentro de estas competencias comunicacionales están el procesar y comunicar información, diseñar y producir ideas gráficas. También se requiere el manejo de una segunda lengua (Martínez y Orozco, 2002), lo cual, gracias a los procesos de globalización y de internacionalización, cobra cada vez más importancia.

c. Competencias generales actitudinales para investigar:

- Competencias sociales como aprender a trabajar en equipo. Esta competencia fortalece la habilidad para trabajar eficazmente con otras personas. El estudiante aprende cuando es sujeto activo del aprendizaje (Pérez, 2012).

- El gusto por la lectura es otra competencia general fundamental para levar a cabo un proceso de investigación es la lectura comprensiva y analítica.

- La curiosidad y motivación por conocer, para plantear preguntas sobre el mundo, elaborar respuestas, diseñar propuestas, exponer los resultados con lenguajes específicos.

- La disciplina, que requiere crear hábitos y tener disposición. para el trabajo y para cumplir con objetivos propuestos. por competencias.

- El interés y el agrado por la tecnología. Se basa en una actitud positiva hacia el uso de las diferentes herramientas tecnológicas.

- Seguridad personal para sustentar lo que se hace frente a otras personas.

Para Mendivelso (2006 como se citó en Leiva, 2013), muchas de estas competencias generales están asociadas a la investigación y se pueden desarrollar en educación básica y media.

a. Competencias específicas cognitivas en investigación: Corresponden al manejo conceptual que los docentes tienen sobre la investigación, y a los procesos que se requieren para llevarla a cabo. Estas competencias se agruparon en cuatro áreas relacionadas con las etapas propias del proceso de investigación. A continuación, se describen:

- Etapa de comprensión científica: esta área incluye competencias relacionadas con el dominar el concepto de ciencia, conocimiento y epistemología de la investigación (Pérez, 2012); identificar 
las etapas del proceso de investigación, reconocer las características de un artículo científico y resolver problemas de investigación.

- Etapa de identificación de la problemática: identificar componentes de la contextualización, comprender la estructura de una pregunta de investigación, dominar el concepto de justificación y distinguir objetivo de actividad (Pérez, 2012).

- Etapa teórica: en esta área se encuentran competencias como identificar la función del marco teórico en una investigación, identificar los componentes de un marco teórico y consultar diferentes fuentes $y$ antecedentes.

- Etapa metodológica: comprender los tipos de investigación, reconocer las técnicas de recolección de datos, identificar los instrumentos de cada técnica, especificar eventos de estudio, definir unidades de estudio, y establecer la diferencia entre población y muestra (Pérez, 2012).

- Etapa analítica: conocer las técnicas de análisis cualitativo y cuantitativo, poseer información sobre las herramientas estadísticas para procesar la información e identificar cómo se formula y comprueba una hipótesis (Pérez, 2012).

- Etapa de socialización: reconocer características de un artículo de investigación, identificar los contenidos del informe final e identificar contenidos de una propuesta (Pérez, 2012), así como comprender métodos de exposición de trabajos (MEN, 2009).

- En el caso de los docentes, una competencia importante, que va más allá de lo estrictamente metodológico es comprender el significado, la importancia y las implicaciones de la investigación educativa en la práctica pedagógica (Federman, Quintero y Munévar, 2001 como se citó en Ollarves y Salguero, 2009).

b. Competencias específicas procedimentales en investigación: Son las habilidades y destrezas relacionadas con el saber hacer, el desempeño y la aplicación de aprendizajes en las etapas del proceso de investigación. Entre otras se encuentran:

- Etapa de comprensión científica: competencias relacionadas con realizar un proceso de investigación completo, escribir un artículo de investigación, resolver problemas de investigación, y aplicar el método científico (Pérez, 2012).

- Etapa de identificación de la problemática: las competencias procedimentales propias de esta etapa son: formular preguntas de investigación, elaborar una justificación, redactar objetivos y especificar los eventos de estudio (Pérez, 2012). También observar, preguntar, analizar, describir contextos y escribir textos acerca de situaciones problemáticas (Federman, Quintero y Munévar 2001 como se citó en Ollarves y Salguero, 2009).

- Etapa teórica: Seleccionar contenidos para elaborar un marco teórico, organizar un esquema conceptual, redactar una fundamentación teórica, especificar eventos de estudio, utilizar líneas de investigación, realizar la justificación de una investigación y aplicar las teorías epistemológicas en la investigación (Pérez, 2012). 
- Etapa metodológica: Las competencias de esta etapa son formular el tipos de investigación correspondiente al estudio, seleccionar unidades de estudio, utilizar técnicas e instrumentos de recolección de información, y formular y comprobar hipótesis (Pérez, 2012). Además, proponer soluciones a los problemas detectados, mediante el uso adecuado de los conceptos y métodos de investigación (Federman, Quintero y Munévar, 2001 como se citó en Ollarves y Salguero, 2009)

- Etapa analítica: analizar datos, formular y comprobar hipótesis de investigación, operacionalizar variables, seleccionar publicaciones científicas, jerarquizar, integrar y recordar datos de una investigación, aplicar las herramientas estadísticas en investigación y analizar un informe de investigación (Pérez, 2012)

- Etapa de divulgación: Perfeccionar las prácticas de la escritura para sistematizar los datos y la información (Federman, Quintero y Munévar, 2001 como se citó en Ollarves y Salguero, 2009). Elaborar artículos de investigación (Pérez, 2012). Realizar exposiciones de los proyectos de investigación (MEN, 2009) y de informes en público (Pérez, 2012).

c. Competencias específicas actitudinales en investigación: Son las inclinaciones afectivas y las cualidades personales, tales como la motivación, la disciplina y el compromiso, entre otros, que favorecen la realización de una investigación. Estas corresponden a:

- Etapa de comprensión científica: incluye competencias relacionadas con la actitud hacia la ciencia, agrado por el conocimiento y epistemología de la investigación: Disposición para aprender a investigar y para investigar; interés por conocer las etapas del proceso de investigación; motivación por leer artículos de investigación y agrado por resolver problemas de investigación (Pérez, 2012).

- Etapa de identificación de la problemática: Esta área abarca competencias como el interés por los componentes de la contextualización, agrado por solucionar problemas del entorno, como interés por comprender la estructura de una pregunta de investigación, disposición para escoger un tema de investigación, honestidad para elaborar una justificación y agrado por analizar objetivos (Pérez, 2012).

- Etapa teórica: Honestidad frente a las fuentes originales de una revisión bibliográfica, disciplina con horarios y cronograma, agrado para aprender sobre metodología de la investigación, compromiso con los problemas del entorno, responsabilidad para elaborar artículos de investigación, orden para estar bien documentado, e interés para aprender sobre técnicas e instrumentos de recolección de información (Pérez, 2012).

- Etapa metodológica: Interés para desarrollar los tipos de investigación, agrado por operacionalizar variables, honestidad al aplicar instrumentos de recolección de datos, agrado por formular tipos de investigación, responsabilidad al seleccionar unidades de estudio, motivación para utilizar técnicas e instrumentos de recolección de información, disposición 
para formular y comprobar hipótesis (Pérez, 2012). También se consideran competencias actitudinales, el compromiso para proponer soluciones a los problemas detectados, mediante el uso adecuado de los conceptos y métodos de investigación (Federman, Quintero y Munévar, 2001 como se citaron en Ollarves y Salguero, 2009).

- Etapa analítica: Motivación para analizar datos y exponer la discusión de resultados de la investigación, compromiso al formular y comprobar hipótesis de investigación, honestidad para jerarquizar, integrar e interpretar datos de una investigación, y responsabilidad al aplicar las herramientas estadísticas en investigación (Pérez, 2012).

- Etapa de divulgación: Motivación por las publicaciones científicas y disciplina para realizar un informe de investigación (Pérez, 2012).

La función del docente en el fortalecimiento de las competencias investigativas es fundamental, porque él constituye un ejemplo de proyecto de vida en el ámbito profesional y social. El docente promueve la expresión de sentimientos y actitudes fundamentadas en valores, además de un pensamiento independiente, disciplinado, creativo, y no solo de transmisión de conocimientos. Esto implica un cambio sustancial en la forma de enseñar y evaluar, lo cual supone un aprendizaje significativo de los aspectos que conciernen a la investigación. Lo anterior da lugar a la apertura de un espacio donde el estudiante pueda elaborar escritos, ensayos, artículos, entre otras cosas. En este aspecto, es clave que el docente comparta sus expectativas, aspiraciones y criterios para realizar la investigación (Pérez, 2012).

\section{MÉTODO}

Esta investigación se desarrolló desde la comprensión holística de la ciencia, la cual según Hurtado (2010) integra los diferentes enfoques de investigación, y por esa razón no se considera un paradigma, sino un sintagma de los modelos epistémicos. Este proceso sintagmático da lugar una definición de investigación donde se reflejan los diferentes modelos epistémicos de manera complementaria, es decir se rompen las contradicciones de los diferentes modelos en la definición de investigación.

El tipo de investigación en la que se enmarcó el presente trabajo fue la descriptiva, que consiste en caracterizar en evento de estudio. El método utilizado fue la holopraxis, como la práctica global de la investigación en sus múltiples dimensiones, según estadios que llevan hasta el nivel de conocimiento propuesto en el objetivo general (Hurtado de Barrera, 2000). Para esta investigación, el método transitó por tres etapas descriptivas. Se utilizó un diseño transeccional (un corte en el tiempo), y de campo, puesto que la indagación se llevó a cabo en el contexto natural donde estaban ocurriendo los hechos, y los datos se obtuvieron de fuentes vivas. La población total fue de 38 docentes, que orientaban para el momento la asignatura de investigación en educación básica y media de las instituciones educativas públicas y privadas del municipio de Sogamoso, Boyacá. No se requirió muestreo, debido a que la población era pequeña, se conocían todos sus integrantes, y era accesible. 
Para medir los conocimientos, RESULTADOS habilidades y disposiciones que poseen los docentes con respecto a metodología de la investigación se utilizó una Prueba de Competencias Investigativas con 86 ítems con puntaje máximo de 261 puntos, y para su interpretación se utilizó una escala transformada de 0 a 50 puntos. Los ítems se agruparon en tres sinergias: competencias cognitivas (28 ítems), competencias procedimentales (13 ítems), y competencias actitudinales (45 ítems). El puntaje bruto de todas las sinergias se transformó a una escala de 50 puntos. La validez por juicio de expertos fue de $0,90, y$ la confiabilidad fue de 0,92.

Para el procesamiento de los datos se utilizó estadística descriptiva, específicamente medianas, frecuencias, porcentajes y sus correspondientes gráficos de caja y sectores. En el Cuadro 1 se muestran los resultados globales de Competencias Investigativas. El puntaje mínimo en una escala de 50 puntos fue de 17,24 y se ubicó en la categoría muy deficiente. El puntaje máximo fue de 43,49 y se ubicó en la categoría excelente.

Cuadro 1. Mediana del grupo de docentes en competencias investigativas

\begin{tabular}{|c|c|c|}
\hline \multicolumn{3}{|c|}{ Competencias investigativas } \\
\hline Mediana & & 30,2700 \\
\hline Mínimo & & 17,24 \\
\hline Máximo & & 43,49 \\
\hline \multirow{3}{*}{ Percentiles } & 25 & 26,2500 \\
\hline & 50 & 30,2700 \\
\hline & 75 & 35,1025 \\
\hline
\end{tabular}

Fuente: Datos obtenidos de la aplicación de instrumentos

La mediana de competencias requeridas para investigar. El gráfico l de investigativas del grupo de docentes fue de 30,27 puntos en la escala de 0 a 50. Este puntaje se ubica en la categoría regular y quiere decir que los docentes que orientan la asignatura de investigación presentan falencias en cuanto a las competencias caja y bigotes muestra que el grupo de docentes es relativamente homogéneo, debido a que el puntaje total de los casos en competencias investigativas se ubica entre 17,24 y 43,49 puntos en la escala de 0 a 50. 


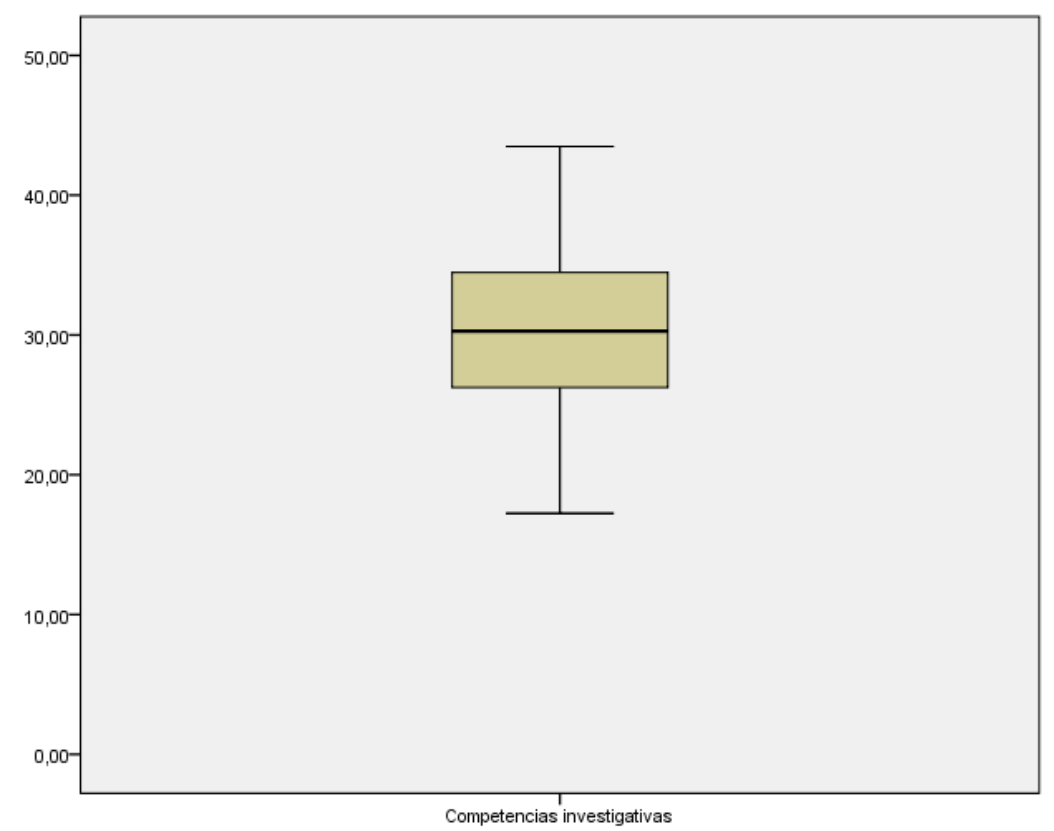

Gráfico 1. Mediana del grupo de docentes en competencias investigativas (Fuente: Datos obtenidos de la aplicación de instrumentos)

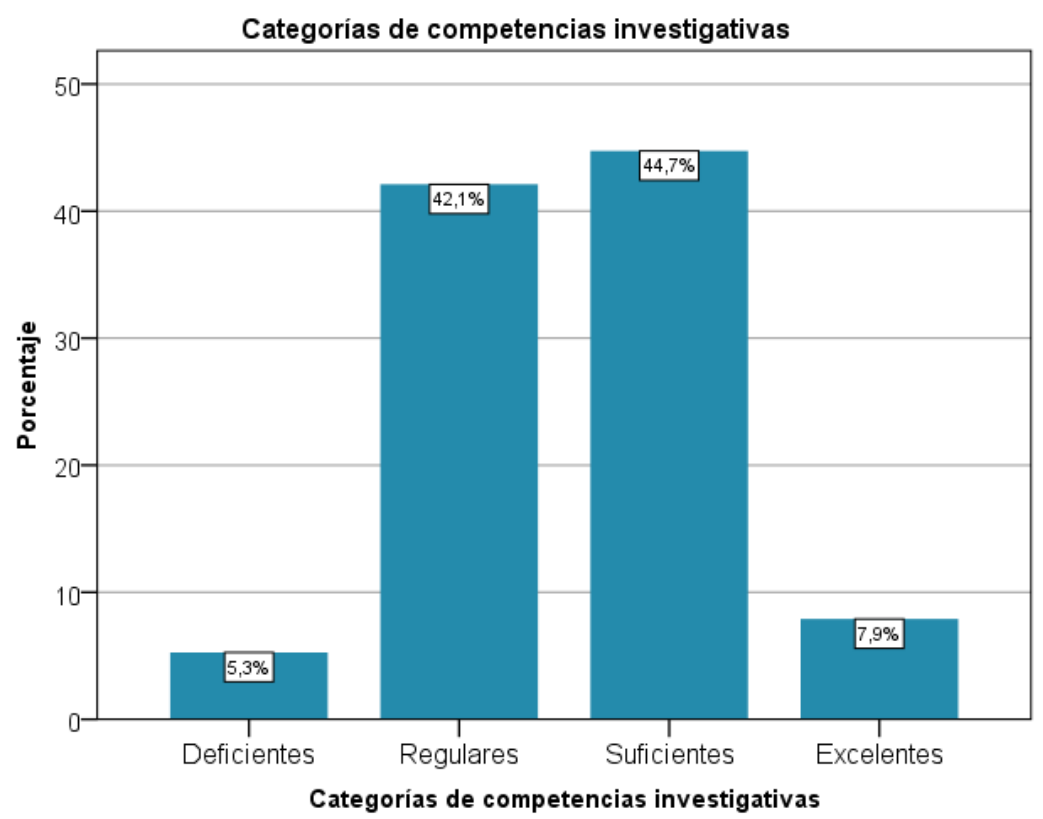

Gráfico 2. Porcentaje de docentes en cada categoría de competencias investigativas. (Fuente: Datos obtenidos de la aplicación de instrumentos)

Los resultados señalados en el gráfico 2 indican que el $47.4 \%$ de los docentes se ubican entre las categorías deficiente y regular, lo que significa que sus competencias para investigar son precarias y no alcanzan un mínimo requerido para hacer una buena investigación. En la categoría suficiente se ubicó el 44,7\% y tan sólo el 7.9\% se ubicó en la categoría en la categoría excelente. Los docentes que alcanzaron esta categoría tienen postgrado, han participado en cursos, talleres $y$ diplomados de profundización en investigación, han publicado artículos, y también pertenecen a una línea de 
investigación. Estas características justifican sus altos puntajes en la prueba de competencias investigativas.

Las competencias investigativas, están conformadas por tres sinergias: competencias cognitivas, competencias procedimentales y competencias actitudinales. En el Cuadro 2 y en el gráfico 3, se analizan éstas.

Cuadro 2. Mediana del grupo de docentes en cada sinergia de competencias investigativas

\begin{tabular}{|c|c|c|c|c|}
\hline & & $\begin{array}{c}\text { Competencias } \\
\text { cognitivas }\end{array}$ & $\begin{array}{c}\text { Competencias } \\
\text { procedimentales }\end{array}$ & Competencias actitudinales \\
\hline Mediana & & 19,7550 & 25,0000 & 35,4150 \\
\hline Mínimo & & 7,26 & 2,63 & 18,33 \\
\hline Máximo & & 29,84 & 42,11 & 50,00 \\
\hline \multirow[t]{3}{*}{ Percentiles } & 25 & 15,9275 & 17,7625 & 30,8300 \\
\hline & 50 & 19,7550 & 25,0000 & 35,4150 \\
\hline & 75 & 22,5800 & 32,2375 & 39,8625 \\
\hline
\end{tabular}

Fuente: Datos obtenidos de la aplicación de instrumentos

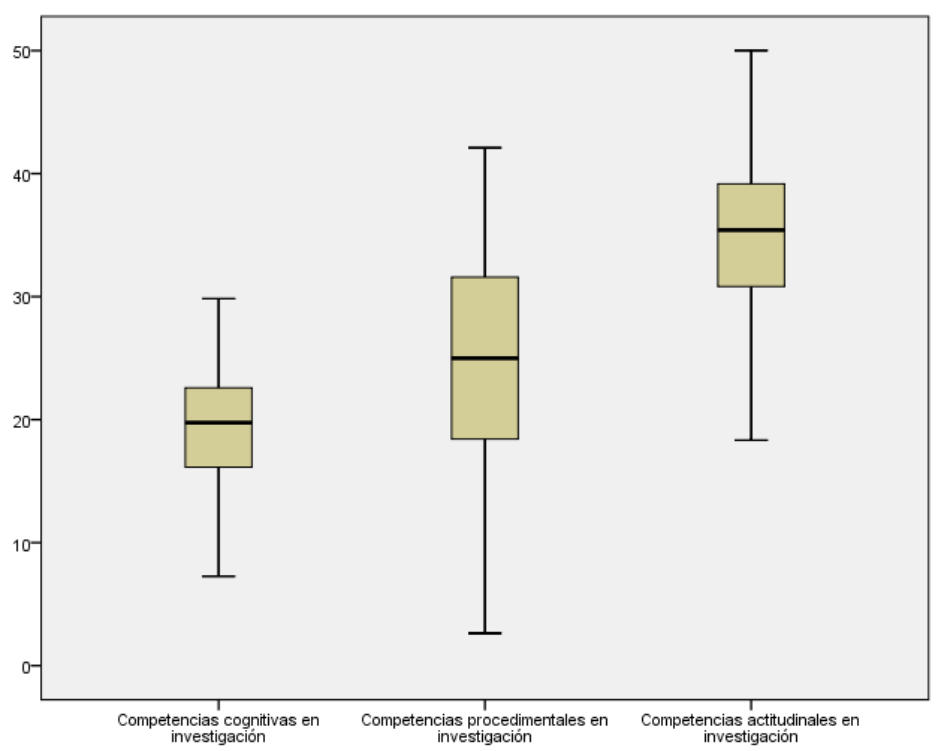

Gráfico 3. Mediana de cada una de las sinergias de competencias investigativas (Fuente: Datos obtenidos de la aplicación de instrumentos)

Curiosamente, la sinergia competencias actitudinales fue la que obtuvo la mediana más alta, lo que quiere decir que, aunque los docentes no tienen conocimientos ni saben realizar completamente una investigación, si tienen una actitud positiva frente a ella. La sinergia más baja fue la sinergia competencias cognitivas y, de las tres, la más heterogénea es competencias procedimentales, mientras que la sinergia más homogénea competencias cognitivas.

En lo que respecta a la descripción de la sinergia competencias cognitivas, el resultado de la mediana, el mínimo, el máximo y los cuartiles, se muestra en el Cuadro 3. 
Cuadro 3. Mediana del grupo de docentes en competencias cognitivas en investigación

\begin{tabular}{|c|c|c|}
\hline Mediana & & 19,7550 \\
\hline Mínimo & & 7,26 \\
\hline Máximo & & 29,84 \\
\hline \multirow[t]{3}{*}{ Percentiles } & 25 & 15,9275 \\
\hline & 50 & 19,7550 \\
\hline & 75 & 22,5800 \\
\hline
\end{tabular}

Fuente: Datos obtenidos de la aplicación de instrumentos

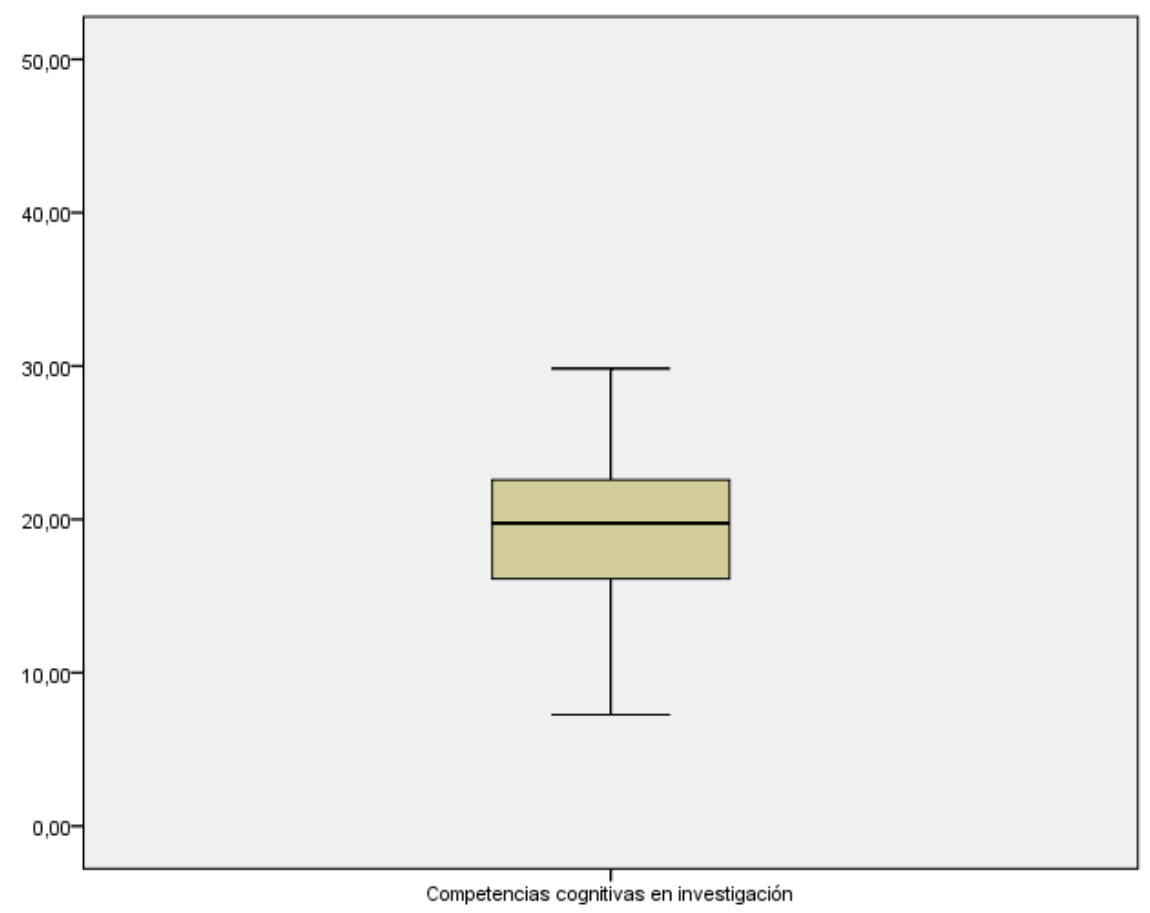

Gráfico 4. Mediana del grupo de docentes en competencias cognitivas (Fuente: Datos obtenidos de la aplicación de instrumentos)

La mediana de competencias cognitivas investigativas del grupo de docentes fue de 19,75 puntos en la escala de 0 a 50, y se ubica en la categoría deficiente. Las competencias cognitivas, corresponden al manejo conceptual que los docentes tienen sobre la investigación y los procesos que se requieren para llevarla a cabo, el resultado anterior significa que los docentes tienen escasa información y poco manejo conceptual acerca de los procesos involucrados en una investigación, lo cual podría afectar su habilidad para orientar la asignatura de investigación. En el gráfico 4 se puede ver que el grupo de docentes es relativamente homogéneo debido a que el puntaje total de los casos se encuentra entre 7,26 y 29,84 puntos en la escala de 50 puntos y el recorrido es de 22,58 puntos.

Los resultados del gráfico 5 revelan que el 50\% de los docentes tienen deficiencias en cuanto al conocimiento en investigación, puesto que el $5,3 \%$ se ubicó en la categoría muy deficiente y el 44,7\% se ubicó en la categoría deficiente. Estos representan la mitad del grupo. El otro 50\% de los docentes se ubicó en la categoría regular, y ninguno alcanzó las categorías suficiente ni excelente. 


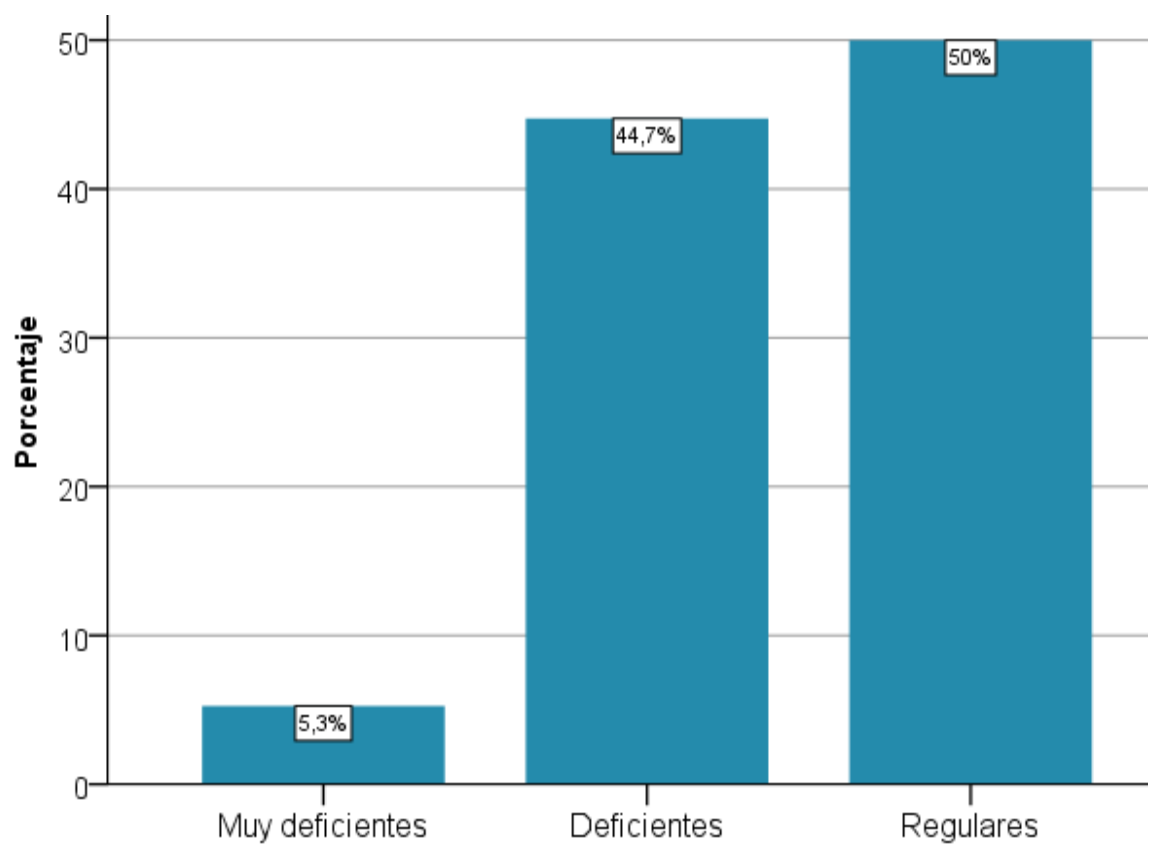

Gráfico 5. Porcentaje de docentes en cada categoría de Competencias Cognitivas. (Fuente: Datos obtenidos de la aplicación de instrumentos)

En el análisis de ítems se evidencia que las competencias cognitivas más desarrolladas en los docentes son: distinguir entre ciencia y tecnología, identificar cualidades del conocimiento, definir investigación, y reconocer su propósito, identificar contenidos de la contextualización, reconocer la media como una técnica de análisis de datos, e identificar resultados de investigación en un artículo.

Las competencias cognitivas en las cuales los docentes tienen más deficiencias, de acuerdo a los resultados, son las siguientes: no identificar preguntas de investigación, ni relacionar las preguntas con el nivel de conocimiento. Tampoco relacionan la pregunta con el tipo de investigación ni con el objetivo, no reconocen las características de una justificación, tienen dificultad para reconocer los verbos relacionados con objetivos de investigación y no relacionan objetivo general con el tipo de estudio. Tampoco reconocen las características de una investigación exploratoria ni de la investigación confirmatoria, no comprenden la relación entre técnicas e instrumentos, y no relaciona la entrevista y la encuesta con sus instrumentos. Lo anterior indica que al parecer lo que más dominan los docentes son las primeras y últimas etapas de un proceso de investigación, concepto, de ciencia, justificación, contextualización, algunos rudimentos de análisis e interpretación de resultados, entre otras. Lo que más se les dificulta es la metodología, validación y elaboración de instrumentos, elaboración de preguntas y objetivos, tipos de investigación etc. Es importante destacar que no tiene sentido analizar resultados que han sido obtenidos con instrumentos mal desarrollados y con escasa validez.

En cuanto a la sinergia competencias procedimentales, la mediana de la sinergia competencias procedimentales fue de 26,32 puntos en la escala de 0 a 50 . Este puntaje se ubica en la categoría regular. 
Cuadro 4. Mediana del grupo de docentes en competencias procedimentales en investigación

Mediana

Mínimo

Máximo

Percentiles
26,3200

42,11

17,7625

25,0000

32,2375

Fuente: Datos obtenidos de la aplicación de instrumentos

Las competencias procedimentales investigativas son las habilidades $y$ destrezas relacionadas con el saber hacer, el desempeño y la aplicación de aprendizajes en las etapas de un proceso de investigación. En este caso, el análisis del resultado indica que los docentes de Sogamoso que orientan la asignatura de investigación presentan algunas carencias para realizar las actividades propias de una investigación. Sin embargo, la mediana de esta sinergia fue mayor que la de competencias cognitivas.

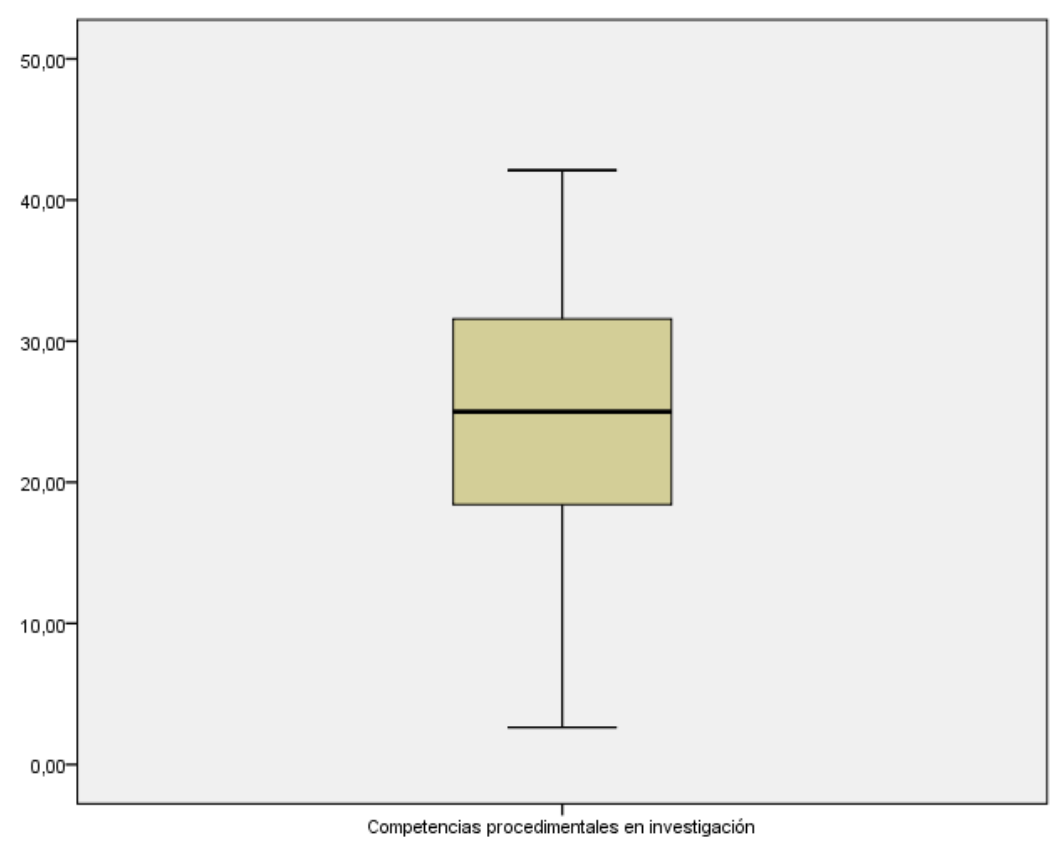

Gráfico 6. Mediana del grupo de docentes en competencias procedimentales en investigación. (Fuente: Datos obtenidos de la aplicación de instrumentos)

En esta sinergia, el grupo de docentes es heterogéneo, debido a que el puntaje total de los casos en competencias procedimentales se encuentra entre 2,63 y 43,11 puntos en la escala de 0 a 50 puntos. El grupo que se encuentra por debajo de la mediana es más heterogéneo que el que se encuentra por encima de la mediana. En el gráfico 7 se puede ver con mayor detalle la distribución del grupo de docentes en las categorías en las frecuencias y porcentajes de la sinergia competencias procedimentales. 


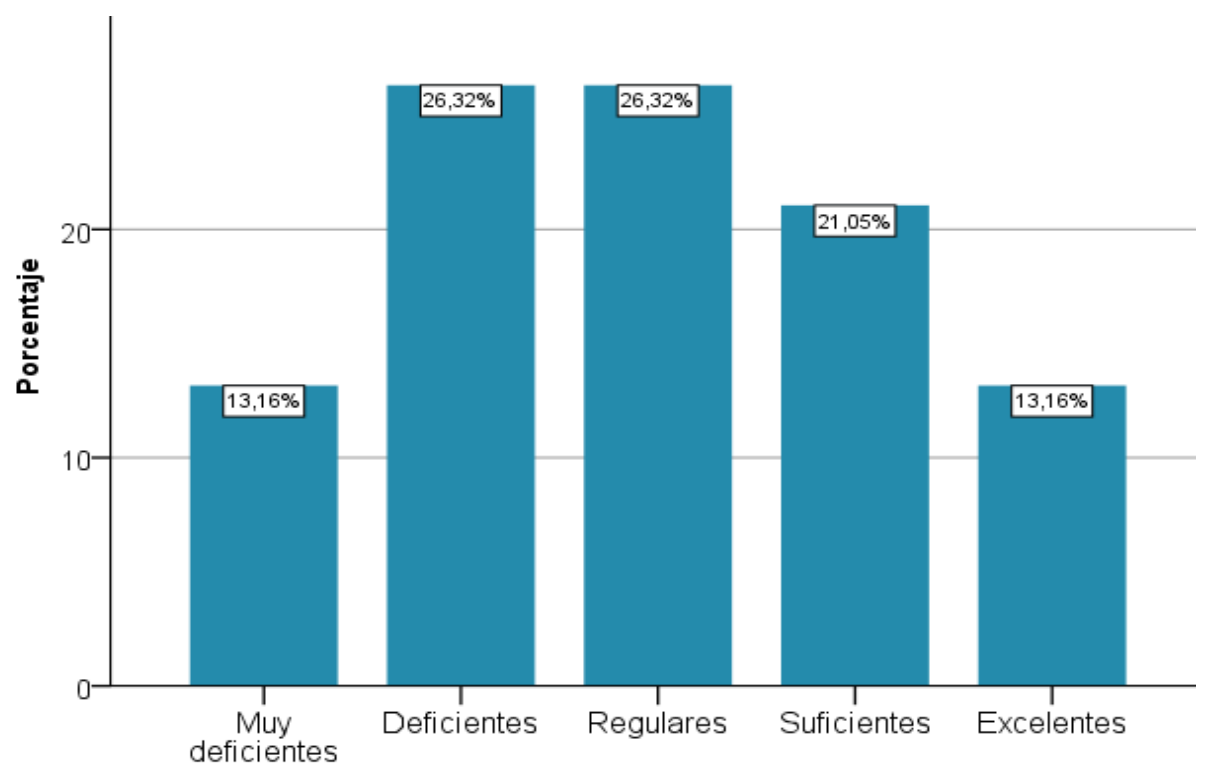

Gráfico 7. Porcentaje de docentes en cada categoría de competencias procedimentales. (Fuente: Datos obtenidos de la aplicación de instrumentos)

Al calcular los resultados de la sinergia competencias procedimentales, los datos analizados del gráfico 7 señalan que el $66,26 \%$ de los casos se ubicaron en la categoría muy deficiente, deficiente y regular, este resultado es preocupante porque esta sinergia tiene que ver con el saber hacer. Esto puede tener consecuencias en el acto de enseñar, en el cual el docente guía y dirige una clase; si no ha realizado procesos de investigación, no logrará trasmitir a los niños cómo elaborar un proceso de investigación. Por otra parte, sólo el $34,21 \%$ de los docentes tiene las habilidades para realizar investigaciones de manera suficiente y excelente. Es esta sinergia no se observaron casos atípicos.

En el análisis de ítems se evidencia que las fortalezas que tienen los docentes en cuanto a competencias procedimentales son: habilidad para extraer la unidad de estudio de una pregunta de investigación, habilidad para extraer la temporalidad de una pregunta de investigación, y escoger la técnica de análisis a partir de una pregunta de investigación. En cuanto a las debilidades que tienen los docentes en las competencias procedimentales se encuentran: no saber elaborar una pregunta de investigación, dificultad para redactar objetivos de una investigación, y no poder describir su tipo de investigación. Un porcentaje muy alto de docentes no sabe formular preguntas ni objetivos de investigación, de lo cual se desprenden otras características principales de un proyecto, tampoco saben formular el tipo de investigación, lo que significa que la mayor falencia que tienen los docentes respecta a la metodología.

En cuanto a competencias actitudinales, el Cuadro 5 muestra la mediana, el máximo, el mínimo y los cuartiles de la sinergia competencias actitudinales para el grupo de docentes. 
Cuadro 5. Mediana del grupo de docentes en competencias actitudinales en investigación

\begin{tabular}{|c|c|c|}
\hline Media & & 35,1349 \\
\hline Mediana & & 35,0000 \\
\hline Mínimo & & 18,33 \\
\hline Máximo & & 50,00 \\
\hline \multirow[t]{3}{*}{ Percentiles } & 25 & 30,8300 \\
\hline & 50 & 35,4150 \\
\hline & 75 & 39,8625 \\
\hline
\end{tabular}

Fuente: Datos obtenidos de la aplicación de instrumentos

La mediana de la sinergia competencias actitudinales, del grupo de los 38 docentes, fue de 35,00 puntos en la escala de 50. Este puntaje se ubica en la categoría suficiente. Esto significa que los docentes tienen buena actitud frente a la investigación, al considerarse las competencias actitudinales como las inclinaciones afectivas y las cualidades personales, tales como la motivación, la disciplina y el compromiso, entre otros, que favorecen la realización de una investigación. Se evidencia en el gráfico 8, que el grupo objeto de estudio es heterogéneo.

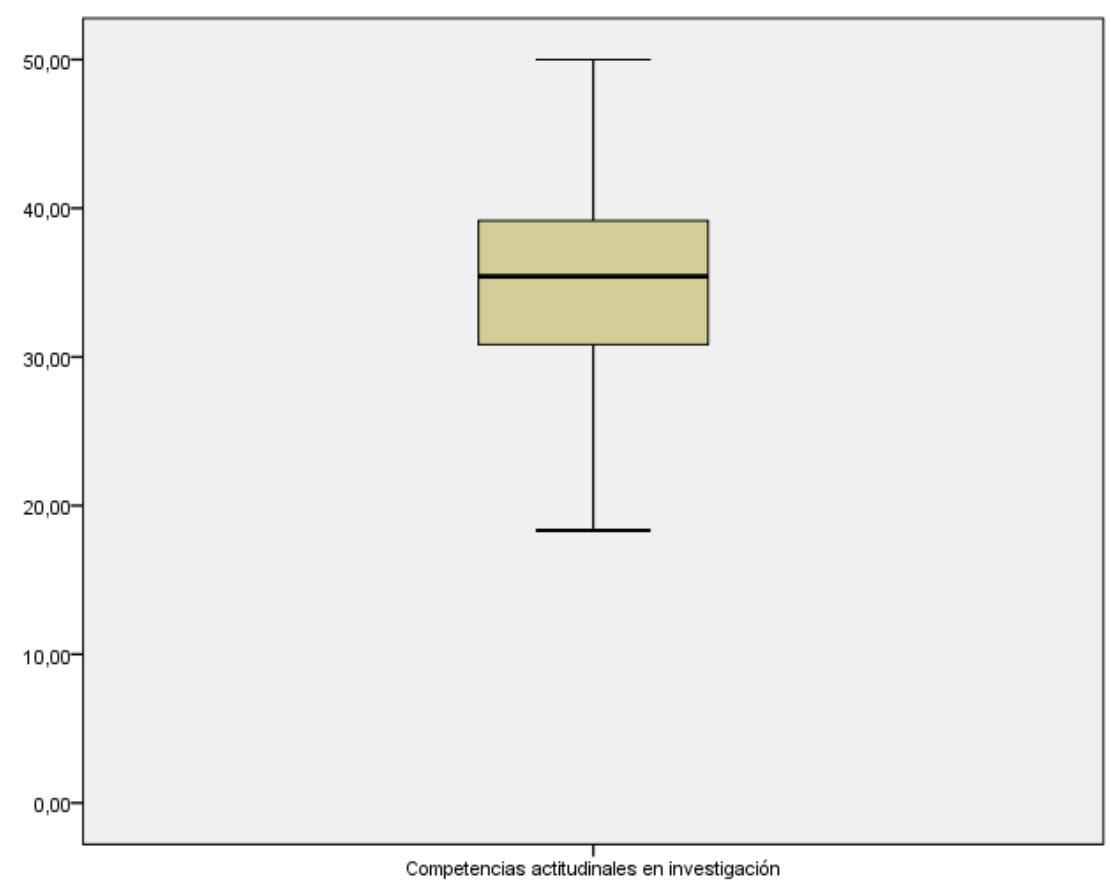

Gráfico 8. Mediana del grupo de docentes en competencias actitudinales en investigación (Fuente: Datos obtenidos de la aplicación de instrumentos)

El 15,8\% de los docentes se ubica en las categorías deficiente y regular, lo que significa que no tienen una buena actitud hacia la investigación. El 60,5\% se ubica en la categoría suficiente, es decir que un alto porcentajes de docentes manifiestan buena actitud para aprender y realizar procesos de investigación. Por último, el 23,7\% se ubica en la categoría de excelente. 


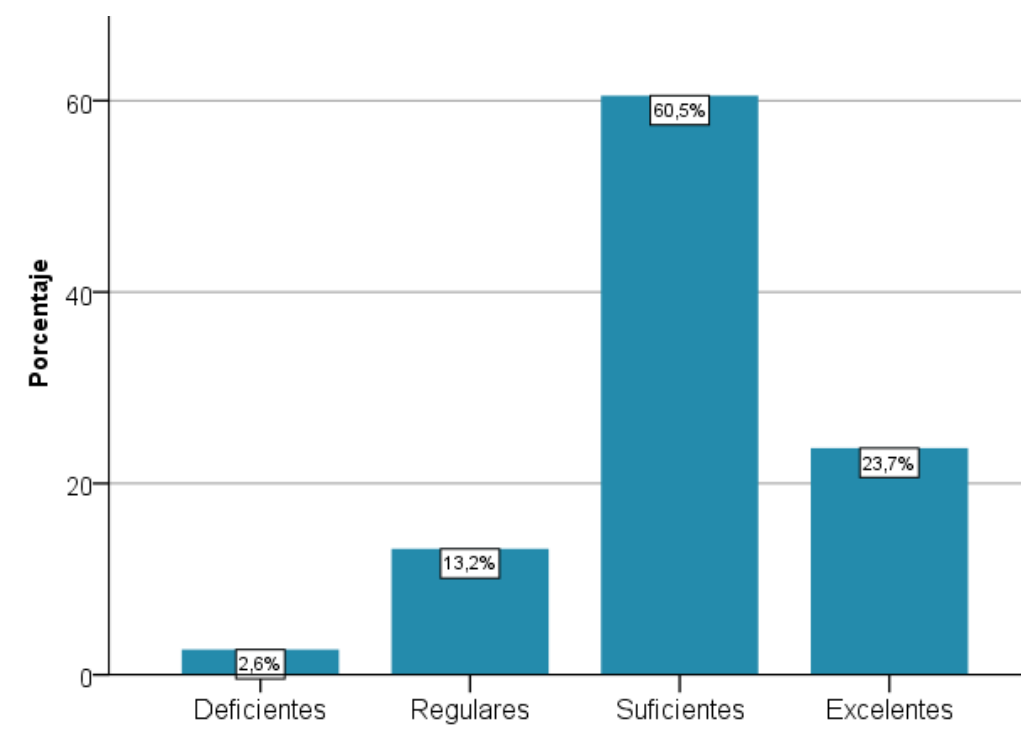

Gráfico 9. Porcentaje de docentes en cada categoría de competencias actitudinales. (Fuente: Datos obtenidos de la aplicación de instrumentos)

Para identificar fortalezas en cada aspecto de la sinergia competencias actitudinales, los ítems se agruparon en cuatro áreas: Proceso de investigación, selección del tema y contextualización, proceso de documentación, y proceso metodológico y analítico. A continuación, se presenta el análisis de cada una.

En cuanto al proceso de investigación, las fortalezas encontradas en las competencias actitudinales fueron: seguridad para defender su tema de investigación, interés en aprovechar tiempo en investigar, cumplir con el trabajo cuando se compromete a terminar una investigación, y disciplina y orden al iniciar una investigación. En lo que respecta al tema y la contextualización, las fortalezas, en cuanto a competencias actitudinales, están representadas por honestidad y responsabilidad con las personas de su entorno, gusto por preguntar, iniciativa y responsabilidad con los problemas de su contexto, e interés por escoger un tema de investigación a partir de su propia experiencia. En general los docentes tienen una buena actitud frente a la selección del tema y la contextualización, les interesan los problemas de su entorno y sienten responsabilidad ante éstos.

Las fortalezas que más se presentan en las competencias actitudinales relacionadas con el proceso de documentación, son: considerar que copiar un texto sin referencia es deshonesto, disciplina para redactar, ser organizado para recoger información, estar dispuesto a desarrollar hábitos de revisión bibliográfica, además de estar motivado para leer artículos y para estar bien documentado. Por otra parte, las competencias actitudinales relacionadas con las actividades metodológicas y analíticas, consisten en la disposición que tienen los docentes para aprender sobre metodología y análisis en investigación.

Como se puede ver, la actitud de los docentes para aprender sobre metodología de la investigación es muy buena. Esto significa que las instituciones educativas podrían ofrecer capacitaciones en investigación y los docentes serían muy receptivos.

\section{CONCLUSIONES}

A partir del estudio realizado, se afinó que los docentes que orientan la asignatura 
de investigación no cuentan con las competencias suficientes para desarrollar un adecuado proceso de investigación. Las competencias cognitivas para investigar fueron las más deficientes. Las competencias procedimentales se ubicaron en la categoría regular, lo que indica que a pesar de tener poco dominio cognitivo de los procesos de investigación, los docentes logran hacer varias de las actividades de las que se requieren probablemente de forma mecánica, sin comprender mucho el sentido de lo que están haciendo. Las competencias investigativas actitudinales fueron las que mejor puntaje obtuvieron, lo que quiere decir que a pesar de que los docentes tienen poco conocimiento y pocas habilidades para desarrollar una investigación, manifiestan una actitud positiva frente a la investigación.

\section{REFERENCIAS}

Aldana de Becerra, G. (2012). La formación investigativa: su pertinencia en pregrado. Revista Virtual Universidad Católica del Norte, (35). 367-379. Recuperado de https://revistavirtual.ucn.edu.co/index.p hp/RevistaUCN/article/view/366

Castillo, M. (1999). Estrategias para la formación de investigadores: una Propuesta en construcción. Recuperado de

http://www.quadernsdigitals.net/datos/ hemeroteca/r_47/nr_503/a_6882/6882.pdf

CEPAL. (29 de noviembre de 2004). América Latina y el Caribe rezagada en investigación y desarrollo [Comunicado de prensa] Recuperado de https:/www.cepal.org/es/comunicados/ america-latina-caribe-rezagadainvestigacion-desarrollo

Colciencias. (2017). Proyectos oferta Colciencias: Jóvenes investigadores e innovadores. Recuperado de https://minciencias.gov.co/sites/default/f iles/upload/paginas/proyecto_oferta_colc iencias_jovenes_investigadores.pdf
Contreras, Z. (2014). Las competencias investigativas. Recuperado de http://www.educando.edu.do/files/1713/3 190/5240/Las_Competencias_Investigativ asl.pdf

García, C. (2009). Formalidad e informalidad en el proceso de aprender a enseñar. Revista de Educación (Madrid). (350). 31-56. Recuperado de http://www.revistaeducacion.educacion. es/re350/re350_02.pdf

Gutiérrez, M. (30 de noviembre de 2014). ¿Maestros investigadores e innovadores? Pesquisa Javeriana. Recuperado de https://www.javeriana.edu.co/pesquisa/ maestros-investigadores-e-innovadores/

Hurtado de Barrera, J. (1999). Diseño de un programa de formación en Metodología de la Investigación para docentes de Postgrado. (Tesis de Maestría). Universidad Central de Venezuela, Venezuela

Hurtado de Barrera, J. (2000). Retos y Alternativas en la Formación de Investigadores, Caracas, Venezuela: Sypal-Fundacite Anzoátegui

Leiva, O. (2013). Formación en Investigación: Una Propuesta de Enseñanza para el Colegio Gimnasio los Pinares. (Tesis de Maestría). Universidad Nacional de Colombia, Medellín, Colombia

Marcelo, C. (2002). Aprender a enseñar para la sociedad del conocimiento. Revista complutense de educación, 12(2), 531-593. Recuperado de https://dialnet.unirioja.es/servlet/articulo ?codigo $=650138$

Martínez, A. y Orozco, J. (2002). Educación superior de alta calidad para interactuar en la sociedad del conocimiento. Recuperado de T. León. (Ed.). Módulo Enfoques y tendencias curriculares. Programa de maestría en Desarrollo Educativo y Social. Bogotá, Colombia: CINDE

Mendivelso, M. (2006). Hacia un aprendizaje comprensivo de la investigación. Bogotá: Colombia. IDEP

Ministerio de Educación Nacional. (2006). Estándares Básicos de Competencias en 
Lenguaje, Matemáticas, Ciencias y Ciudadanas. Recuperado de https://www.mineducacion.gov.co/162l/ articles-340021_recurso_l.pdf

Ministerio de Educación Nacional. (2009). Desarrollo infantil y competencia en la primera infancia. Recuperado de https:/www.mineducacion.gov.co/prim erainfancia/1739/articles-178053_archivo_ PDF_libro_desarrolloinfantil.pdf

Ministerio de Educación Nacional. (2010). Estándares Básicos de Competencias en Lenguaje, Matemáticas, Ciencias y Ciudadanas. Recuperado de https://www.mineducacion.gov.co/1621/ articles 340021_recurso_l.pdf

Ollarves, Y. y Salguero, L. (2009). Una propuesta de competencias investigativas para los docentes universitarios. Laurus, 15(30). 118-137. Recuperado de https://www.redalyc.org/pdf/761/761206 51006.pdf

Pérez, M. (2012). Fortalecimiento de las competencias investigativas en el contexto de la educación superior en Colombia. Revista de investigaciones UNAD, 11 (1). 9-34. Recuperado de https://doi.org/10.22490/25391887.770

Quiroga, M., Arredondo, E., Cafena, D. y Merino, C. (2014). Desarrollo de competencias científicas en las primeras edades: el Explora Conicyt de Chile. Educ. Educ. 17 (2), 237-253. Doi 10.5294/edu.2014.17.2.2

Restrepo, B. (2004). Formación Investigativa e investigación Formativa: Acepciones $y$ Operacionalización de esta última y Contraste con la Investigación Científica en Sentido
Estricto. Recuperado de http://planmaestroinv.udistrital.edu.co/d ocumentos/PMICI-UD/InvestigacionFor mativa/Formaci $\%$ C3\%B3n\%20Investigat iva $\% 20$ e\%20investigaci\%C3\%B3n\%20F ormativa.pdf

RICYT. (2018). El estado de la ciencia principales indicadores de ciencia y tecnología iberoamericana/interamericana. Recuperado de

https://www.oei.es/historico/divulgacio ncientifica/?el-estado-de-la-ciencia-princ ipales-indicadores-de-ciencia-ytecnologia

Tacca, D. (2011). El "nuevo" enfoque pedagógico: las competencias. Investigación educativa, 15 (28). 163-185. Recuperado de https://revistasinvestigacion.unmsm.edu. pe/index.php/educa/article/view/5426/4 673

Tobón, S. (2006). Aspectos básicos de la formación basada en competencias. Recuperado de https://maristas.org.mx/gestion/web/doc tos/aspectos_basicos_formacion_compet encias.pdf

Tobón, S. (2007). El enfoque complejo de las competencias y el diseño curricular por ciclos propedéuticos. Acción pedagógica, 16(1). 14-28. Recuperado de https://dialnet.unirioja.es/servlet/articulo ?codigo $=2968540$

Zamora, N. (2014). La formación investigativa de los estudiantes: un problema aún por resolver. Escenarios, 12(2), 76-85. DOI: https://doi.org/10.15665/esc.v12i2.316 